\title{
Information Requirements of Health Systems as Drug Purchasers: Does the FDA Have a Role in Setting Evidentiary Standards?
}

\author{
Paul C. Langley
}

\begin{abstract}
The objective of this article is to consider the information needs of drug purchasers; whether the Food and Drug Administration (FDA) should have a role in regulating information on the characteristics and cost-outcomes impact of new pharmaceutical products to health care purchasers; and the requirements in the new FDA Modernization Act of 1997 for "competent and reliable scientific evidence."

Market-mediated advertising claims are compared to regulated market claims and the role of the market in potentially limiting the flow of deceptive
\end{abstract}

information. The author concludes that the FDA should not attempt to micromanage information flow in promotion and marketing campaigns, but should consider the Federal Trade Commission approach to deceptive advertising with possible inputs in gray areas from an expert panel.

KEYWORDS: FDA, Regulation, Economic information, Pharmacoeconomics

J Managed Care Pharm 1998: 593-598

$\mathrm{T}$ he purpose of this paper is to consider whether the Food and Drug Administration (FDA) plays a legitimate and useful role in regulating the promotional and marketing activities of pharmaceutical manufacturers by establishing evidentiary standards for pharmacoeconomic evaluations that focus on managed care and other health systems as drug purchasers.

In late 1997, the U.S. Congress passed the FDA Modernization Act of 1997, which has given this issue new importance. Section 114 of this act (entitled Health Care Economic Information) amends the previous 1992 legislation by adding to Section 502(a) (21 U.S.C. 352(a)) the statement, "Health care economic information provided to a formulary committee, or other similar entity, in the course of the committee carrying out its responsibilities for the selection of drugs... shall not be considered to be false or misleading... if the information directly relates to an indication approved .... and is based on competent and reliable scientific evidence."

Two issues are involved here: 1) should the FDA be involved in the regulation of health care economic information (as defined by the legislation ${ }^{1}$ ); and 2) if we believe the FDA should be involved, what should its responsibilities be? Some would argue that the FDA does have a role, but only to the extent that it operates, as does the Federal Trade Commission (FTC), to "defer deceptive claims that harm consumers (the users of such information) but not to interfere unnecessarily with the dissemination of truthful, nondeceptive information." Such a role would require the FDA to eschew attempts to micromanage pharmacoeconomic studies by attempting to control the flow of information and by setting standards for marketing and promotion that may do more harm than good.

The FDA's role must recognize the power of the market-

place to police the activities of pharmaceutical manufacturers.
PAUL C. LANGLEY, PH.D., is Professor, Department of Pharmacy Practice, School of Pharmacy, University of Colorado Health Sciences Center, Denver, CO.

Copyright $(\subset) 1998$ Academy of Managed Care Pharmacy, Inc. All rights reserved. 
The reasoning underlying this position holds that the market for pharmaceutical products is highly competitive. As a result, there is a strong presumption that we can rely on market forces to ensure that the types of studies that pharmaceutical manufacturers undertake, and the claims they make for particular products, will meet the professional standards found in the economic evaluation literature and, in consequence, are unlikely to be deceptive. Whereas advertising can be deceptive, legal requirements imposed on advertising have the potential to discourage truthful claims about the economic aspects of pharmaceutical products. However, as is pointed out below, a highly competitive environment for drug manufacturers in specific disease or therapy areas is likely to be equally, if not more, effective. Advertising has the potential to encourage competition, to facilitate the acceptance of new products, and to ensure that consumers of health care make effective choices. Relying on market forces to drive evidentiary standards is more likely to ensure that such studies meet the information needs of drug purchasers.

As far as the FDA is concerned, one principal concern lies in defining the informational needs of drug purchasers. Should the FDA take these into account? What information does the FDA consider appropriate for formulary decisions? Until the FDA articulates its position, it is difficult to see how we can square the emphasis the FDA places on experimentally based evidence with the perceived information needs of managed care systems and other drug purchasers. However, to be fair, few, if any, of those working in the area of health care evaluations seem to have considered these information needs and how manufacturers might address them in their choice of study design and analytical perspective. This is all the more surprising when we consider the attention that pharmacoeconomics literature gives to the role of guidelines in formulary submissions ${ }^{3}$ and to the promotion of reference standards. ${ }^{4}$ Although mandatory submission guidelines are found in only a few countries with national health care systems, such as Australia, Canada, and Switzerland, in the United States, managed care has taken the initiative in developing submission guidelines. ${ }^{5}$ Unfortunately, at this time, few seem to be aware of the existence of these guidelines and the perspective they give on the information needs of managed care in formulary decision making.

\section{TRADITIONAL VERSUS SYSTEMS PERSPECTIVES IN ECONOMIC EVALUATIONS}

One of the more puzzling and unfortunate features of pharmacoeconomic analysis is the extent to which many, if not most, of those working in the area are wedded to what may be described as the traditional evaluation paradigm. The hallmark of the traditional paradigm is the assumption that the information needs of health care purchasers can be met by the estimation of cost-outcomes ratios. These ratios typically are grounded in a simple decision-model framework that compares drug
A to drug B; in many cases, the analysis is based directly on clinical trial results. This focus is most clearly expressed in the revised November 1995 Australian Guidelines, in which all submissions are required to provide a preliminary economic evaluation based on trial results. ${ }^{6}$

The FDA also embraces this traditional perspective. Consider the statement that "effectiveness elements of costeffectiveness claims must be based on adequate and well controlled studies." Although this might be interpreted as an FDA endorsement of naturalistic randomized-controlled trials (in using the term "effectiveness" rather than "efficacious"), in promotion and marketing terms it means that the pharmaceutical manufacturers should base any claims for cost-effectiveness on the traditional randomized-controlled trial or on models that extrapolate from those trials. The status of attempts to model drug impacts with data drawn from a variety of data sources, or to use retrospective analyses as the basis for promotional and marketing claims, is unknown. Indeed, it is important to point out that in FDA legislation and regulation the terms efficacy and effectiveness are used interchangeably.

The essence of the systems approach is to recognize that any evaluation of the potential or projected impact of a new therapy must take into account the treating environment, the impact of introducing that therapy on the distribution of patients among therapy options, and the consequences of these factors on the costs of delivering therapy to that treating population and on their outcomes profile. The systems approach, which takes explicit account of the information needs of drug purchasers, is the perspective found in guidelines documents published by managed care in the United States. ${ }^{8.9}$ These guidelines, which follow closely a template proposed by Langley and Sullivan, ask pharmaceutical manufacturers to report on: 1) the safety, efficacy, and effectiveness of their product relative to existing medications; and 2) a pharmacoeconomic evaluation, involving outcomes modeling, which includes an assessment of the impact of the new product on the outcomes profile of the treating population, across all options in a disease or therapy area, and on the direct costs of treatment within that therapy area. ${ }^{10}$

In the Regence Washington Health guidelines, manufacturers are asked to consider two scenarios: first, an evaluation of the current costs of providing treatment in that therapy area; and, second, an estimate of the impact of introducing the new product once "a new equilibrium of resource utilization and outcomes has been established." The guidelines note that the latter scenario requires estimates of patterns and rates of drug substitution and the redistribution of patients between treatment pathways. The characteristics of the treating population are taken into account. The impact of introducing a new drug into a treatment area also can take account of budget constraints, as well as of health care intervention programs that may be in place or may be suggested to optimize the effectiveness of the particular drug or therapy. 
Of course, when a health system has guidelines in place, the issue is not one of promotion and marketing, because the pharmaceutical manufacturer is responding to a request for information to support a formulary decision. However, our concern lies with the status of promotion and marketing activities by pharmaceutical manufacturers that may attempt to use a systems-based evaluation modeling framework to explore the impact of introducing a new product for a representative managed care purchaser, or to report on modeling exercises undertaken for specific purchasers or health systems.

Clearly, the systems perspective and its information requirements represent a major shift in analytical focus, one that is far removed from the traditional, clinically based evaluation paradigm. Not only are the information needs of the systems approach more demanding, but also the need to model the net cost and outcomes impact within a therapy or disease area creates a major challenge for pharmaceutical manufacturers and for those evaluating a systems-based model or an actual formulary submission. One way of differentiating the traditional and systems perspectives in an advertising context is to see the traditional approach as supporting efficacy claims and the systems approach as focused on effectiveness claims-that is, claims based on the anticipated impact of a product in a real-world treating environment. We can conceive of traditional approaches that extrapolate from clinical trials and attempt to include, within a modeling framework, modifications of clinical endpoints and cost estimates to reflect actual treating environments (e.g., compliance and discontinuation behavior). However, the challenge facing health care evaluations is to translate clinical findings into claims that reflect the characteristics of the treating population, their behavior, and the organizational framework within which health care is delivered. If, for example, the manufacturer believes that compliance behavior and the nature of contracts between health systems and health care providers will significantly modify trial-based claims for clinical efficacy, then these points should be made explicit to potential drug purchasers. It seems pointless and deceiving to sweep these issues under the carpet by setting regulatory standards that exclude attempts to evaluate the impact of these factors simply because the regulatory agency considers that such evaluations fail to meet standards for scientific rigor.

This emphasis on a systems perspective does not mean that claims for particular products might not be based on other analytical frameworks. There are, for example, a number of nonexperimental approaches to drug impact assessment that might form the basis for marketing and promotion claims. Retrospective studies using claims databases might be commissioned to evaluate cost savings associated with drug interchanges or the impact of particular patterns of compliance behavior on claims for cost-effectiveness. Indeed, considerable resources have been allocated by economists to resolving (or at least understanding) the technical issues associated with generating unbiased estimates of the impact of social programs on income and employment outcomes. There seems no reason why these techniques could not be applied to issues of retrospective drug impact assessment, forming the basis for marketing and promotion activities.

The systems perspective also may be contrasted to what may be described as the component approach to cost containment. Under this approach, the accounting and reporting framework within health systems is compartmentalized, with budgetary targets set for often arbitrarily defined cost centers. These cost centers may cut across disease or therapy area boundaries and bear no resemblance to any meaningful activity-accounting framework that encompasses both pharmacy and medical costs in disease or treatment areas. In many health systems a major cost center is the formulary, where targets and criteria for drug selection reflect only drug costs. The possible adverse impact of drug restrictions on nondrug health care costs and the possibility that overall health care delivery costs may increase following restrictions on drug purchases are not taken into account. There appears to be ample evidence to support this latter proposition-evidence that is either ignored or not seen as relevant by financial managers. ${ }^{11}$

Indeed, the so-called restricted versus open formulary debate may be seen as a variant of the component management argument. In its most widely publicized version, this debate focuses on an observed negative statistical association between the openness of a formulary and the utilization of ambulatory care visits, hospital admissions, and prescription drugs. ${ }^{12}$ Although the link between the openness of a formulary and the costs of health care delivery is never fully articulated (the argument being essentially observational), a number of commentators have interpreted these findings as supporting the case for an open formulary in which there is (apparently) no need for a prior pharmacoeconomic evaluation of drug therapies, because all products are immediately admitted. Such a position has an obvious appeal to pharmaceutical manufacturers, although it overlooks the role of formularies in health systems and their potential contribution to the delivery of health care.

Needless to say, this is a highly dubious argument and one many commentators believe is built on a flimsy methodological and empirical base..$^{13}$ One key issue is how we define and interpret, in quantitative terms, an index of the degree of openness of a formulary. After all, the absence of particular drug products from a formulary may be due to either an a priori exclusion based on acquisition cost considerations or to an ex post facto exclusion decision following a systematic clinical and economic evaluation of therapy options.

A more serious concern lies in the potential for regulatory authorities arbitrarily to restrict the flow of information to health care decision makers, or (possibly inadvertently) to countenance evidentiary standards that conflict with the information needs of those purchasers. Both these scenarios could have an adverse impact on the cost effectiveness of health care delivery. It does not automatically follow that health care 
systems simply will subscribe to component-cost management in drug evaluations and formulary listing in the absence of this information; however, it could mean that the type of information that manufacturers are allowed to provide is inappropriate for that treating environment. Regulation must not stand in the way of meeting the information requirements of purchasers; the market must be allowed to respond to those needs.

\section{CLINICAL TRIALS AND EVIDENTIARY REQUIREMENTS}

For those coming from a clinical background, the randomized-controlled trial is typically seen as the evidentiary gold standard in claims for drug efficacy and safety. Pharmacoeconomic evaluations, which usually involve piggy-backing resource utilization and outcomes measures onto an established clinical protocol, are judged on this standard. External validity, which in this context meets the information needs of drug purchasers, takes a back seat. This is not to deny that a number of commentators have argued for naturalistic trials as a basis for greater external validity and as a counterweight to modeling, which many continue to view with suspicion. ${ }^{14}$ However, as these approaches still accept the traditional evaluative framework, they go only a small part of the way toward meeting the information needs of drug purchasers. ${ }^{15}$

Clinical trials are coming under increasing scrutiny. Although they typically are defended for their ability to "eliminate the potential for selection bias to affect mean-impact estimates," Heckman and Smith conclude that "the existing literature overstates many of the other arguments in their favor."16 The authors identify five principal criticisms of social experiments: the failure to provide answers to many questions of interest to policy makers; the failure to provide useful information on the distributional impact of programs; the presence of randomization bias; the presence of institutional limitations on social experiments; and the presence of substitution bias. Although not all of these criticisms apply with equal strength to clinical trials, the authors argue that "there is a sizable divergence between the theoretical capabilities of experiments based on random assignment and the practical results of such evaluations."

More important-and this is a key point to note-Heckman and Smith argue that many who advocate the experimental approach to program evaluation "ignore promising developments in the theory and practice of nonexperimental evaluations." The authors also consider the contribution of "self-contained black-box experimental evaluations" to the general body of social science knowledge. They maintain that such evaluations "pose a serious threat to the accumulation of knowledge about the behavior of persons and institutions." Indeed, because "they are not conducted within a behaviorally coherent framework of analysis, the evidence from experiments does not accumulate." These are important criticisms which, although levied in an evaluation of training programs for disadvantaged groups, apply with equal force to attempts to evaluate the impact of new and existing drug therapies on treating populations. ${ }^{17}$ Clinical trials ignore the context within which programs or therapies are to be delivered, the divergent characteristics of treating populations, and the distribution of outcomes and strategies open for patient management; they must be seen only as a first step in therapy evaluation. Nonexperimental retrospective drug evaluation designs, which might utilize claims databases, present major challenges but may be equally important in the promotional and marketing activities undertaken by pharmaceutical manufacturers.

In short, from the perspective of a managed care group; the evidence provided by clinical trials may be (and typically is) of limited utility. Unless trial results can be translated to a particular treatment setting or at least factored into an impact assessment model, claims for the superiority of one drug over another based on a particular trial protocol are likely to be ignored. Such claims accepted at their face value may mislead an uncritical formulary committee as to their impact in a disease or therapy area.

\section{SUBSTANTIATION AND VERIFICATION}

The question of translating clinical results into practice settings raises further issues of substantiation and verification. The term "substantiation" can be considered from two perspectives: 1) as a legal or regulatory requirement; and 2) as a procedure for evaluating experimentally and nonexperimentally based claims. The new act takes a legal or regulatory perspective on substantiation. In evaluating health care information, the FDA will accept information provided as part of a promotional or marketing activity if it is based on competent and reliable scientific evidence. The FTC, in invited comments, has been at pains to discourage the FDA from putting advertised claims into a regulatory straitjacket. It argues, first and foremost, that the types of economic claims that are likely to be made cover a wide range. They would include claims based on both experimental and nonexperimental study designs, as well as those based on the modeling of disease interventions for target populations. It should be emphasized that substantiation also is posssible when a drug manufacturer takes a system perspective to predict the impact of a particular new product on the costs and outcomes within a representative treating environment, or on an environment modeled to allow such cost and impact predictions for alternative treating scenarios. If a pharmaceutical manufacturer can substantiate the choice of assumptions, including key parameter values, then there seems to be no reason why claims cannot be based on such an approach.

Cost-effectiveness claims that pharmaceutical manufacturers make for their products should be verifiable-that is, manufacturers should couch their claims so that predictions about the impact of the drug in a given treating environment can be empirically assessed. In the United States it is clearly possible, given the availability of pharmacy and medical claims data, to 
evaluate claims about the rate of market penetration of a drug, patient switching patterns, and the cost implications of introducing a new product. Unfortunately, traditional trial-based economic evaluations or evaluations based on extrapolations from clinical trials, with their focus on cost-outcomes ratios as decision variables, cannot generate testable predictions. If a pharmaceutical manufacturer bases promotion and marketing activities on such models, there is no way to substantiate the veracity of these claims. This is not a question of misleading or incomplete information, but reflects the fact that traditional pharmacoeconomic evaluation has its roots in experimental, randomized-controlled trials rather than in the nonexperimental design of mainstream economics, and attempts to model and predict the impact of new drug therapies in given treating environments.

Systems models, however, have the potential to generate testable predictions. Because these models focus on the impact of introducing a new product on the costs and outcomes for a defined population in a given treatment environment, predictions about the magnitude of this impact can be made and such claims verified by monitoring medical and pharmacy claims (where the latter, it is hoped, would include clinical markers).

\section{PROMOTION, MARKETING, AND INDICATION}

The FDA will draw the line at allowing pharmaceutical manufacturers to make claims for the off-label use of a product. This places the prospective purchaser of a drug in a difficult situation. Although there are, quite clearly, concerns about claims for off-label use-situations in which a drug has not met FDA requirements for safety and efficacy-there is equally clear evidence that many drugs have substantial potential for off-label uses. Prospective purchasers need to know about these patterns of off-label use, which have direct implications for budget impacts and patient outcomes. Purchasers also need to know what prospective applications the manufacturer will make for expanding indications for a drug. There seems to be no reason why drug manufacturers could not disseminate such information as part of their marketing activities. But because manufacturers are unlikely to do this, it is up to the drug purchaser, as part of formulary submission guidelines, to request such information and factor it into purchasing decisions.

\section{DECEPTION, INCOMPLETE INFORMATION, AND MARKET COMPETITION}

For the FTC, a claim for a product is considered to be deceptive "if it contains a material representation or omission of fact that is likely to mislead consumers acting reasonably under the circumstances." This is typically considered from the perspective of the audience. Whether or not a particular claim is considered deceptive involves consideration of a number of factors "relevant to the benefits and costs of substantiating a particular claim... [including] type of claim...consequences of a false claim... benefits of a truthful claim" and the degree of substantiation that experts in the field might consider reasonable.

In the case of pharmacoeconomic evaluations and claims made for pharmaceutical products, there is not only a wide range of claims that can be made (which may be based on a "selective" use of the scientific evidence), but the claims made can vary in terms of data sources, study design (where clinical trials are one option), analysis techniques target audience, and organizational or institutional context of the analysis. Any attempt to set standards for deceptive advertising not only must take this diversity into account (e.g., evaluative ability of the target audience), but also must ensure that substantiation rules do not stifle the flow of useful information. Claims based on randomized clinical trials may be judged deceptive if such factors as biases inherent in clinical trials, compliance behavior, and misdiagnoses by physicians are not taken into account.

The fact that information is incomplete does not mean that the claim made is necessarily deceptive, although a deliberate attempt to suppress critical information would be construed as such. As Ippolito points out, three features of the marketplace are important in considering incomplete information: competition, background information, and consumers. ${ }^{18}$ In competitive markets, a marketing claim that emphasizes one aspect of a product is likely to be countered by competitors if it is successful in switching sales. Also, purchasers of a product do not rely simply on advertised claims. There is a range of other data sources that can be drawn on in making a purchasing decision. Finally, consumers (which would include both prospective patients and medical practitioners) are well aware that advertising is likely to emphasize the positive features of a product and thus will make appropriate allowances. Indeed, in the case of medical practitioners, we have an expert purchasing group well qualified to make a clinical evaluation, but possibly less well placed to make a pharmacoeconomic one. However, we should not overlook pharmacy and therapeutics committees, which, as agents for health systems and medical practitioners, also have a key role in evaluating claims for particular products.

In short, the competitive nature of the health care market is probably the single most important reason for believing that, even without FDA or FTC regulation, the amount of deceptive advertising is likely to be minimal. Companies that believe their competitors are guilty of deception are likely to respond rapidly, whereas companies that feel they are losing market share because they are not meeting the information needs of purchasers will adjust their promotion and marketing activities. Although whistle-blowing is, presumably, not to be encouraged, it is clear that complaints would be made to the FDA or a nominated adjudicator if it were felt that competitors were engaged in deceptive practices.

\section{CONCLUSIONS}

We face an interesting dilemma in any attempt to regulate the form and content of pharmacoeconomic evaluations. If the 
FDA enshrines traditional trial-based pharmacoeconomic evaluation as the only form of evaluation capable of meeting the criteria of competent and reliable scientific evidence within a given indication, the information needs of drug purchasers are unlikely to be met.

As has been argued, the use of the word "scientific" would appear at face value to be redundant. If competent and reliable evidence would reasonably be judged acceptable by an independent observer in the appropriate field, then there is no need to debate the scientific nature of that evidence. Although the multidisciplinary nature of health care evaluations will admit to some disputation regarding the standards accepted by those from different disciplines, there seems to be sufficient consensus.

The FDA has argued that all "effectiveness elements of costeffectiveness claims must be based on adequate and well-controlled studies." This is not a position to which the majority of health economists would subscribe. Unless we resolve this issue and admit a wider range of studies-studies that take explicit account of the information needs of health care purchasers-then we face a bleak future in the provision of useful and truthful information to health care purchasers. It is difficult to see how a regulatory body can set standards that differentiate between traditional and systems perspectives, study designs, techniques, and analyses used to support promotional and marketing activities, and those that would be disallowed. Of course, the FDA might attempt to enlist the contribution of health economists and convene an expert committee of leading health economists to advise on a set of standards. There is a precedent for this in the expert committee convened by the National Oceanic and Atmospheric Administration to report on willingness-to-pay techniques. ${ }^{19}$ However, given the nature and range of economic analyses that can be applied to pharmaceutical evaluations (where willingness-to-pay evaluations are only one type), it seems doubtful that a set of standards can support such a differentiation-not to mention the legal interpretations that may be placed upon them. The only practical options open to the FDA, if it wishes to introduce such standards, are either to insist on a narrowly defined set of standards (e.g., evaluations must be experimentally based and report only on the outcomes of randomized pharmacoeconomic trials) or to establish a pharmacoeconomic review committee that makes recommendations on proposed and completed economic evaluations that manufacturers and others may wish to use as part of a promotion and marketing strategy.

To an economist, this is clearly a second-best solution. If our ultimate objective is to ensure that pharmaceutical manufacturers are not discouraged from producing pharmacoeconomic studies that meet the needs of drug purchasers in varied treating environments, then the FTC approach might appear the most appropriate. It still could involve an expert committee to review studies that are considered to be deceptive, but it would not be involved in policing initial proposals for pharmacoeconomic studies or reviewing those undertaken for promotion and marketing by pharmaceutical manufacturers.
If the FDA takes the view that the phrase "competent and reliable scientific evidence" must be interpreted as evidence based on randomized-controlled experiments, then we are back to square one. If the FDA takes a more eclectic position and refers to accepted standards of professional practice within the appropriate discipline, in this case health care economics, then we can look forward to entering a more information-rich environment-and one that will present some interesting challenges not only to pharmaceutical manufacturers but also to health economists and health care purchasers. Unless a more eclectic regulatory position is taken, it is most unlikely that the discipline of pharmacoeconomics will become anything more than an exercise in generating clinical trial-based cost-outcomes ratios.

\section{$\Delta \quad$ References}

1. U.S. FDA Modernization Act 1997, Section 114

2. Public hearings before the Department of Health and Human Services, Food and Drug Administration. Washington, DC: Federal Trade Commission Bureaus of Consumer Protection and Economics; January 16, 1996, Docket No. $95 \mathrm{~N}-0228$ : 5 .

3. Langley PC. Pharmacoeconomics: achieving gold standards. London: Financial Times Health Care Publications, 1997.

4. Gold MR, et al. Cost-effectiveness in health and medicine. New York: Oxford University Press, 1997

5. Langley PC, Martin RE. Managed care guidelines for the economic evaluation of pharmaceuticals. Am J Managed Care 1997; 3: 733-41.

6. Langley PC. The November 1995 revised Australian guidelines for the economic evaluation of pharmaceuticals. PharmacoEconomics 1996; 9: 341-52.

7. 60 Federal Register 41, 892; August 14, 1995.

8. Langley PC, Martin RE. Guidelines for formulary submissions. Rancho Cordova, CA: Foundation Health and Integrated Pharmaceutical Services, October 1996.

9. Regence Washington Health Pharmacy Services. Guidelines for the submission of clinical and economic data supporting formulary consideration. Seattle: Regence Washington Health, 1997.

10. Langley PC, Sullivan SD. Pharmacoeconomic evaluations: guidelines for drug purchasers. J Managed Care Pharm, 1996; 2(6): 671-77.

11. Soumerai SB, et al. Effects of limiting Medicaid drug-reimbursement benefits on the use of psychotropic agents and acute mental health services by patients with schizophrenia. New England J Medicine 1994; 49: 2207-10. 12. Horn SD, et al. Intended and unintended consequences of HMO cost-containment strategies: results from the managed care outcomes project. Am J Managed Care 1996; 2(3): 253-64

13. Vaczek D. Formularies: a debate flares. Pharmacy Times 1996(July): 53 54 .

14. Drummond MF, Spilker B, ed. The future of pharmacoeconomics in quality of life and pharmacoeconomics in clinical trials. Philadelphia: LippincottRaven, 1996

15. Langley PC. The future of pharmacoeconomics: a comment. Clinical Therapeutics 1997; 19(1): 762-69.

16. Heckman JJ, Smith JA. Assessing the case for social experiments. J Economic Perspectives 1995; 9(2): 85-1 i 0 .

17. Friedlander $D$, et al. Evaluating government training programs for the economically disadvantaged. J Economic Literature 1997; 35(4): 1809-55. 18. Ippolito $P$. Issues in regulating economic claims in health-care markets. Conference Paper, Policy Issues in Pharmaceutical Cost-Effectiveness Research, American Enterprise Institute, Washington, DC; Nov 1996

19. Portney PR. The contingent valuation debate: why economists should care. J Economic Perspectives 1994; 8(4): 3-18 